\title{
A Study on the Acceleration of Coronal Mass Ejections
}

\author{
Jie Zhang ${ }^{1}$ \\ ${ }^{1}$ School of Computational Sciences, George Mason University, \\ 4400 University Drive, MSN 5C3, Fairfax, VA 22030, USA \\ email: jiez@scs.gmu.edu
}

\begin{abstract}
.
We present a statistical study on the acceleration of CMEs. This study is based on $23 \mathrm{CME}$ events best observed by SOHO LASCO/C1 coronagraph, which observes the inner corona from 1.1 to $3.0 R_{S}$. The kinematic evolution of a CME has a distinct acceleration phase that mainly takes place in the inner corona. We find that the acceleration duration distribution ranges from 10 to $1100 \mathrm{~min}$ with a median (average) value at $54 \mathrm{~min}$ (169 min). The acceleration magnitude distribution ranges from $6 \mathrm{~m} \mathrm{~s}^{-2}$ to $947 \mathrm{~m} \mathrm{~s}^{-2}$ with a median (average) value at $209 \mathrm{~m} \mathrm{~s}^{-2}$ $\left(280 \mathrm{~m} \mathrm{~s}^{-2}\right)$. We also find a good correlation between CME acceleration magnitude A (in unit of $\mathrm{m} \mathrm{s}^{-2}$ ) and acceleration duration $\mathrm{T}$ (in unit of min), which can be simply described as $\mathrm{A}=10000 / \mathrm{T}$.
\end{abstract}

Keywords. Sun: corona, Sun: coronal mass ejection (CMEs)

\section{Introduction}

CME is a phenomenon observed as a systematic motion of a large scale coronal structure. It has been found that a CME's full kinematic evolution may undergo three distinct phases: an initiation phase of slow rising, a major acceleration phase of fast velocity increasing, and finally a propagation phase with minor velocity changing (Zhang et al. 2001). The first two phases mainly occur in the inner corona (e.g., $<2.0 R_{S}$ ), while the third phase is largely observed in the outer corona (e.g., $>2.0 R_{S}$ ) by traditional white light coronagraphs.

Based on thousands of CMEs observed by Solwind (Howard et al. 1985), SMM (Hundhausen et al. 1994) and SOHO/LASCO (Moon et al.2002, Yashiro et al. 2004), it has been consistently found that CME velocity distribution in the outer corona ranges from about $50 \mathrm{~km} \mathrm{~s}^{-1}$ to $2600 \mathrm{~km} \mathrm{~s}^{-1}$; the average velocity in the distribution is about $400 \mathrm{~km} \mathrm{~s}^{-1}$ with a median velocity at $350 \mathrm{~km} \mathrm{~s}^{-1}$ and a peak velocity at $300 \mathrm{~km} \mathrm{~s}^{-1}$.

On the other hand, the statistical knowledge about CME acceleration in the inner corona remains relatively poor. St.Cyr et al (1999) made a statistical study on CME acceleration using $46 \mathrm{CME}$ features observed by ground-based Mauna Loa K-coronameter in the inner corona combined with SMM observations in the outer corona. They found that acceleration distribution ranged from $-218 \mathrm{~m} \mathrm{~s}^{-2}$ to $3270 \mathrm{~m} \mathrm{~s}^{-2}$ with an average (median) value at $264 \mathrm{~m} \mathrm{~s}^{-2}\left(44 \mathrm{~m} \mathrm{~s}^{-2}\right)$. This might be the only statistical study on CME acceleration so far. However, they did not distinguish acceleration phase from subsequent propagation phase in their calculation. In this paper, we present a study on CME acceleration based on advanced LASCO observations. 

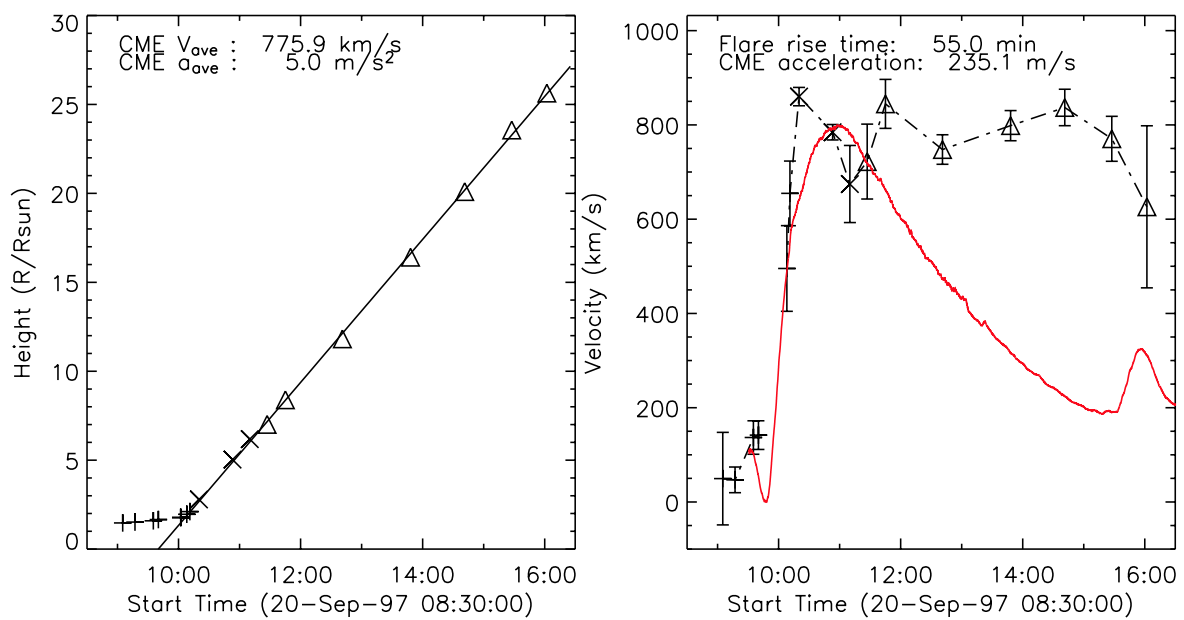

Figure 1. Kinematic plots for the CME on 1997 Sep. 20. The left panel shows the CME height-time measurements from LASCO C1(plus), C2(cross) and C3(triangle) coronagraphs. The straight line is a linear fit to the $\mathrm{H}-\mathrm{T}$ in the outer corona. The right panel shows the CME velocity-time evolution (indicated by dotted-line-connected symbols with error bars) . The velocity is obtained by applying piece-wise numeric derivative to the $\mathrm{H}$ - $\mathrm{T}$ measurement. The smooth solid curve denotes the intensity-time profile of the associated soft X-ray flare.

\section{Observation and method}

We make uses of SOHO LASCO C1, C2 and C3 coronagraph observations, which have fields of view from 1.1 to 3.0, 2.2 to 6.0 and 4.0 to $30.0 R_{S}$, respectively (Brueckner et al. 1995). The combined field of view of LASCO enables one to investigate the full kinematic evolution of CMEs. The $\mathrm{C} 1$ coronagraph provides key observations of CME acceleration in the inner corona. While C2 and C3 are typical white-light coronagraphs that observe Thomson-scattered photospheric light from free electrons in the corona, C1 observes the coronal emission lines (forbidden optical lines) from highly ionized ions. LASCO C1 has successful observations for a period of two and half year from 1996 Jan. to 1998 June; it unfortunately became out of function after the SOHO interruption in 1998. We have explored all C1 images obtained during that period, in total about 100,000 image. We are able to find $101 \mathrm{C} 1 \mathrm{CMEs}$. A C1 CME is that at least one CME leading edge is seen in $\mathrm{C} 1$. As a comparison, there are about $860 \mathrm{C} 2 \mathrm{CMEs}$ during the same period. The much smaller number of $\mathrm{C} 1 \mathrm{CMEs}$ is simply due to the fact that the stray light level is much higher in $\mathrm{C} 1$ because of its closeness to the bright solar disk. As a consequence, most $\mathrm{C} 1$ CMEs are strong CMEs originated close to the limb. Please refer to Zhang et al. (2004) for a more detailed discussion about $\mathrm{C} 1$ observations. Because a full CME kinematic evolution may undergo three distinct phases: initiation phase, acceleration phase and propagation phase, a realistic measurement of CME acceleration should be limited to the time range of acceleration phase only.

We illustrate the method of calculating CME acceleration in Figure 1. The CME occurred on 1997 Sep. 20, observed by C1, C2 and C3 with 8, 3 and 7 snapshot images, respectively. The left panel shows the height-time $(\mathrm{H}-\mathrm{T})$ plot of the $\mathrm{CME}$ from direct measurement of CME leading edge in the images. The right panel shows the CME velocity-time ( $\mathrm{V}-\mathrm{T})$, derived from first-order numerical derivative of the $\mathrm{H}-\mathrm{T}$ measurement. Numerical derivative is essentially a piece-wise fitting to obtain local velocity at each time based on a small set of neighboring H-T points. This is in contrast to the nominal functional fitting, which is essentially a global method to obtain the average velocity 
(first order polynomial fitting) and average acceleration (second order polynomial fitting) of CME over the entire time range, without differentiating different kinematic phases.

CME acceleration is obtained by examining the $\mathrm{V}-\mathrm{T}$ plot (see Figure 1, right panel), Based on the plot, the acceleration started at about 9:40 UT, at which time the CME already had a velocity of about $120 \mathrm{~km} \mathrm{~s}^{-1}$. Note that this CME started to rise at about 09:05 UT, but velocity kept low till 09:40 UT; this phase is so called initiation phase. The CME velocity peaked at about 10:20 UT, or about 40 minute after the onset of fast acceleration; this 40 minute is the acceleration duration of the CME acceleration phase. Because of the limitation of $\mathrm{C} 1$ observing cadence, which is irregular from a few minute to 20 minute, the acceleration duration thus obtained may subject to an uncertainty up to 20 minute. Another way to obtain the acceleration duration is to use the flare rise time as proxy. It has been found that CME major acceleration occurs largely during the rise phase of associated soft X-ray flare (Zhang et al. 2001, Zhang et al. 2004). While there may be time difference between $\mathrm{CME}$ and flare timing, the difference largely falls into the uncertainty in CME measurement. We calculate the CME acceleration magnitude to be $235 \mathrm{~m} \mathrm{~s}^{-2}$, by simply adopting the flare rise time 55 minut as the CME acceleration duration and adopting the CME average velocity $776 \mathrm{~km} \mathrm{~s}^{-1}$ in the $\mathrm{C} 2 / \mathrm{C} 3$ field of view as the CME velocity increasing during the acceleration phase. Note that thus calculated CME acceleration magnitude has an uncertainty, which may range from $10 \%$ for well observed events to up to $50 \%$ for relatively poor observed events.

To carry out the statistical study on CME acceleration, we choose 23 best observed C1 CME events among the $101 \mathrm{C} 1 \mathrm{CMEs}$ in our list. Each of these 23 events has at least $3 \mathrm{CME}$ leading edges seen in $\mathrm{C} 1$, as well as good observations in $\mathrm{C} 2$ and $\mathrm{C} 3$. We use the method illustrated above to calculate CME acceleration magnitude, which is based on the obtained CME acceleration duration and CME velocity increasing during this duration. This relatively large set of 23 events further demonstrate that there is a temporal coincidence rule between CME acceleration and flare flux rise. Therefore, we use flare rise time as CME acceleration duration when a CME is assocaited with a flare. When a CME is not associated with a flare (e.g., gradual event), or when a CME is originated from backside of the Sun with the possible accompanied flare not observable, we directly use V-T plot to estimate CME acceleration time.

\section{Results}

The CME acceleration distribution for the 23 events is shown in Figure 2 (cross symbols), along with average acceleration in the outer corona (plus symbols). Apparently, CME acceleration has a wide distribution from event to event. For the 23 events, the maximum value is $947 \mathrm{~m} \mathrm{~s}^{-2}$ and the minimum value is $6 \mathrm{~m} \mathrm{~s}^{-2}$, with a medium value at $209 \mathrm{~m} \mathrm{~s}^{-2}$ and an average value at $280 \mathrm{~m} \mathrm{~s}^{-2}$. These acceleration parameters should be treated as the CME true acceleration, which is measured during the acceleration phase only. These numbers are much different from that of CME acceleration measured in the outer corona. The acceleration distribution in the outer corona is within a much narrower range, with a minimum value at $-13 \mathrm{~m} \mathrm{~s}^{-2}$ (or deceleration), a maximum value at 40 $\mathrm{m} \mathrm{s}^{-2}$, the medium value is $3 \mathrm{~m} \mathrm{~s}^{-2}$, and the average value at $4 \mathrm{~m} \mathrm{~s}^{-2}$. CME has a much smaller acceleration magnitude in the outer corona, in other words a more or less constant speed; this is so called propagation phase.

Another interesting result is shown in Figure 3. It seems that there is a linear anticorrelation between CME acceleration magnitude and CME acceleration duration (in logarithm scale). Both CME acceleration magnitude and duration vary by more than 2 orders of magnitude. The acceleration duration ranges from 10 to 1100 minute with 


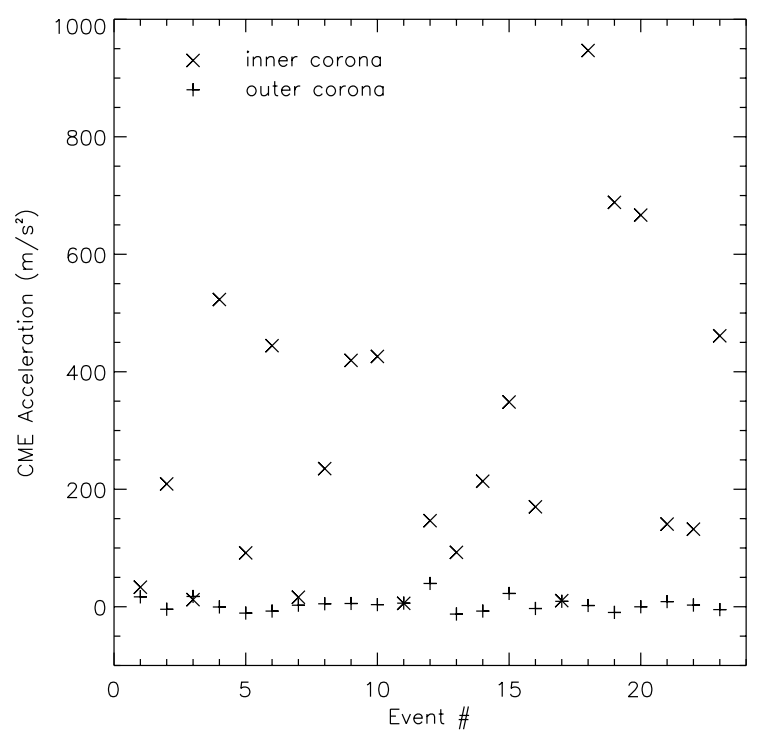

Figure 2. The comparison of CME acceleration in the acceleration phase (mainly in the inner corona, cross symbols) and in the outer corona (or in the propagation phase, plus symbols), for the 23 events with the best LASCO/C1 observations. The events are displayed in time order from left to right along the $\mathrm{X}$-axis.

a median (average) value at 54 minute (170 minute), while the acceleration magnitude distribution ranges from $6 \mathrm{~m} \mathrm{~s}^{-2}$ to $947 \mathrm{~m} \mathrm{~s}^{-2}$. The linear correlation is prominent across this broad range of distribution. Nevertheless, there is large scattering at local level, e.g, acceleration magnitude may vary by a factor of 5 for those CMEs with acceleration duration of tens of minutes. In a general sense, the weaker the CME acceleration magnitude, the longer the CME acceleration duration. For the cases of gradual CMEs with acceleration magnitude of less than $10 \mathrm{~m} \mathrm{~s}^{-2}$, the acceleration duration can be as long as 1000 minute. On the other hand, the very impulsive events with acceleration duration less than 10 minute, the acceleration magnitude can be as high as $900 \mathrm{~m} \mathrm{~s}^{-2}$. The linear fitting to the correlation yields a relation of acceleration magnitude $\mathrm{A}$ (in unit of $\mathrm{m} \mathrm{s}^{-2}$ ) and acceleration duration $\mathrm{T}$ (in unit of minute), simply described as $\mathrm{A}=10000 / \mathrm{T}$.

\section{Discussion}

A CME's kinematic evolution has a distinct acceleration phase. The acceleration magnitude during this phase can vary significantly from event to event. For the 23 best observed C1 CMEs events from 1996 Jan. to 1998 June, the acceleration distribution is found to be from $6 \mathrm{~m} \mathrm{~s}^{-2}$ to $947 \mathrm{~m} \mathrm{~s}^{-2}$. If considering all other events, e.g., extremely impulsive events, the acceleration magnitude distribution may extend to even higher values. The CME on 1997 Nov. 6 (associated with an X9.4 flare) was found to have an acceleration about $7300 \mathrm{~m} \mathrm{~s}^{-2}$ (Zhang et al. 2001). Alexander, Metcalf \& Nitta (2002) reported that a CME-associated X-ray ejecta had a peak acceleration of about $4800 \mathrm{~m} \mathrm{~s}^{-2}$ based on YOHKOH SXT observations. Gallagher,Lawrence \& Dennis (2003) found a CME-associated EUV ejection feature reaching a peak acceleration of about $1500 \mathrm{~m} \mathrm{~s}^{-2}$ based on TRACE observations. Therefore, it seems that there exists a continuous distribution of CME acceleration from extremely impulsive events down to extremely gradual events. However, it is still an open question whether there exists a single peak or multiple 


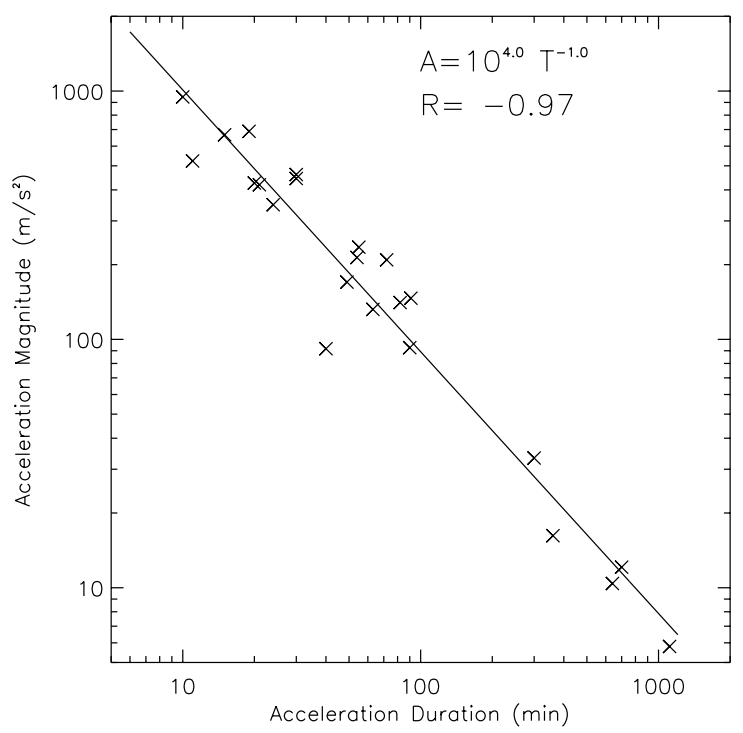

Figure 3. The scattering plot of CME acceleration magnitude versus CME acceleration duration measured in CME's acceleration phase, for the 23 events with the best LASCO/C1 observations. Note that there is a good linear correlation (correlation coefficient -0.97) between the two parameters

peaks on the acceleration distribution, which is related to the issue of CME classifications. To answer this question, a statistical study on a much larger number of events is needed.

\section{Acknowledgements}

J. Zhang is supported by NASA grants NAG5-11874 and 200730, and NSF SHINE grant ATM-0203226.

\section{References}

Alexander, D., Metcalf, T.R., Nitta, N.V. 2002 Geophys. Res. Lett. 29, No.10, 41

Brueckner, G.E., et al. 1995 Sol. Phys. 162, 357

Howard, R.A., Sheeley, N.R., Jr., Michels, D.J., Koomen, M.J. 1985 J. Geophys. Res. 90, 8173

Hundhausen, A.J., Burkepile, J.T., St. Cyr, O.C 1994. J. Geophys. Res. 99, 6543

Gallagher, P.T., Lawrence, G.R., Dennis, B.R. 2003 ApJ 588, L53

Moon, Y.-J., Choe, G.S., Wang, H., Park, Y.D., Gopalswamy, N., Yang, G., Yashiro, S. 2002 ApJ 581, 694

St. Cyr, O.C., Burkepile, J.T., Hundhausen, A.J., and Lecinski, A. R. 1999 J. Geophys. Res. 104, No. A6, 12493

Yashiro, S., Gopalswamy, N., Michalek, G., St. Cyr, O.C., Plunkett, S.P., Rich, N.B., Howard, R.A. 2004, J. Geophys. Res. 109, A7, 7105

Zhang, J., Dere, K.P., Howard, R.A., Kundu, M.R., and White, S.M. 2001, ApJ 559, 452

Zhang, J., Dere, K.P., Howard, R.A., Vourlidas, A. 2004, ApJ 604, 420

\section{Discussion}

MunOz: How were the events used in the analysis selected? 
ZHANG: The two dozen events used in the analysis are those events having very good observations in LASCO C1. Specifically, at least three CME snapshot image are observed by $\mathrm{C} 1$. This is necessary for further analysis of velocity evolution in the inner corona.

GOPAlSwARY: Comment: You talked about "true" acceleration in the inner corona $\left(<2 R_{s}\right)$ and "residual" acceleration in the outer corona. This is some what misleading because the propelling and retarding forces act at all heliocentric distance. Propelling, retarding (gravity and drag) may have different magnitudes at different heliocentric distance, but the resultant is always "true".

ZHANG: The "true" acceleration I used refers to the phase of rapid velocity increase till the peak velocity. But I agree with your comment. The point is that the acceleration, in the inner corona, is much different from that in the outer corona. 